\title{
SIMULATION INPUT MODELS: RELATIONSHIPS AMONG EIGHTY UNIVARIATE DISTRIBUTIONS DISPLAYED IN A MATRIX FORMAT
}

\author{
Wheyming Tina Song \\ Yi-Chun Chen \\ Department of Industrial Engineering and Engineering Management \\ National Tsing Hua University, No.101, Section 2, Kuang-Fu Road \\ Hsinchu, 30013, TAIWAN
}

\begin{abstract}
This paper presents a user-friendly display, in a ten-by-eight matrix format, of a collection of 80 univariate distributions and their interrelationships. A simplified five-by-five matrix, showing only 25 families, is designed for student use. These relationships provide rapid access to information that must otherwise be found through a time-consuming search of numerous sources.
\end{abstract}

\section{INTRODUCTION}

Univariate probability distributions are important because they provide the underpinnings of many of the engineering models that are used in practice, for example, normal (Gaussian) for communication systems and quality control, exponential for queueing, and Weibull for reliability. Univariate distributions are taught in most probability and statistics courses in schools of business, engineering, and science.

A figure illustrating the relationships among univariate distributions is useful for showing how distributions correspond to one another. Nakagawa and Yoda (1977), Leemis (1986), and Leemis and McQueston (2008) offer diagrams of 76 relations among univariate distributions. These diagrams are difficult to use, however, as required distributions are not easy to locate. We believe a diagram that displays the information in a matrix format is far more useful. Song (2005) organized 25 and 35 often-used distributions in the form of a matrix to facilitate a logical display, thereby enhancing ease of location.

This paper improves upon these previous efforts by offering 80 relations in one user-friendly figure, which retains the logical ordering provided by a matrix format. Moreover, we have corrected some errors in Figure 1 of Leemis and McQueston (2008). Trying to locate the pairs corresponding to these errors will clearly illustrate the need for the matrix form we introduce in this paper.

Some useful references for studying univariate distributions are Johnson and Kotz (1970), Johnson, Kotz, and Balakrishnan (1995), Patil, Boswell, and Ratnaparkhi (1985), Patil et al. (1985), Evans, Hastings, and Peacock (2000), Kotz and van Dorp (2004), Devroye (2006), and Dudewicz and Karian (2009). None of these books, however, provide all of the simple relationships shown in this article.

\section{A TEN-BY-EIGHT MATRIX}

Figure 1 illustrates 80 univariate distributions, including 21 discrete and 59 continuous distributions. The discrete distributions are displayed in rectangular boxes in the top three rows, with the third row containing both discrete and continuous distributions. The row and column numbers are labeled along the left-hand side and the top of Figure 1, respectively. Each distribution is then indexed in the form $\mathrm{RiC} j$, where $i=1,2, \ldots, 10$ and $j=1,2, \ldots, 8$. For example, Normal is indexed as $\mathrm{R} 4 \mathrm{C} 2$. The probability mass and density functions are summarized in Appendix A, where distributions are ordered 
alphabetically. The index number shown in the parenthesis beside the name of each distribution allows users to easily find it in Figure 1.

\subsection{Notation}

In Figure 1, the distribution name and parameters are shown in each matrix cell. The range for continuous distributions is also listed in the last row of each cell. Due to the limited space, the range for discrete distribution is not shown. The parameters adopted satisfy the following conventions:

- $\quad N, K, n$ and $k$ are integers;

- $0 \leq p \leq 1$

- $a$ is the minimum; (and also a location parameter)

- $\quad b$ is the maximum; (and also a location parameter)

- $\quad$ is the expected value; (and also a location parameter)

- $\quad$ is the standard deviation;

- $m$ is either the median or mode;

- $\quad$, with or without subscripts, is the location parameter;

- $\quad$ is the scale parameter;

- $\quad$ is the non-centrality parameter.

Notation for shape parameters is more complicated. We always use (with or without subscripts) to denote the shape parameter. For example, the following are shape parameters: the mode parameter $m$ in the Triangular $(a, m, b)$, the integer $k$ in the $\operatorname{Erlang}(k, \quad)$, the degrees of freedom in the ChiSquared( $)$, and the degrees of freedom 1 and 2 in the $F(1,2)$. There are more exceptions.

\subsection{Relations}

Relations between distributions are indicated with a dashed, solid, or mixed dash-solid lines. Dashed lines show asymptotic relations, solid lines show transformations or special cases, and mixed dashsolid lines show mixture relations. For convenience, we use "source" and "target" to label two related distributions. That is, each line shows a relation from its "source" distribution to its "target" distribution. The random variable $X$ is used for all source distributions. Sometimes, when space is limited, two lines are combined into one with two arrows; the relation is then placed closer to the target distribution. For example, there is a solid line with two arrows connecting the Normal (R4C2) and the Standard Normal (R4C3). The relation $(X-) /$, close to the right arrow, indicates that the source distribution is the Normal (R4C2) and the target distribution is the Standard Normal (R4C3). The other relation, $\quad+X$, which is close to the left arrow, indicates the reverse.

In Figure 1, if more than one random variable is involved to form a transformation, the relationship between these random variables is denoted by "iid" (independent and identically distributed) and "indep" (independent). For example, the relation between the Geometric (R2C2) and the Negative Binomial (R3C2) is marked with " $k_{i=1}^{k} X_{i}$, iid", which indicates that the sum of $k$ iid Geometric random variables yields a Negative Binomial random variable.

The transformation relationships in Figure 1 can be combined to form other relationships. For instance, a path from the Standard Normal (R4C3) to the Chi-Squared (R4C4) to the Gamma (R6C4) to the Exponential (R8C3) indicates that the random variable $X_{1}^{2}+X_{2}^{2}$ has the Exponential $(=2)$ distribution if $X_{1}$ and $X_{2}$ are independent Standard Normal random variables.

There is a special relationship between the Standard Uniform (R8C4) and any continuous distribution. That is, $F_{X}(X) \sim \operatorname{Uniform}(0,1)$, where $F_{X}$ is the cumulative distribution function (cdf) of any continuous random variable $X$. Therefore, a connection with the relation $F_{X}(X)$ could be drawn from any continuous distribution to the Standard Uniform. Connections with the relation $F_{X}^{-1}(U)$, where $U \sim$ Uniform $(0,1)$, could be drawn from the Standard Uniform to any distribution. The random variable $F_{X}^{-1}(U)$ is known as the inverse-cdf transformation (Devroye, 2006; Gentle, 2003; and Schmeiser, 1980). When space allows, we show a connection indicating the inverse cdf between the 
Standard Uniform $U$ and other distributions. For example, we show the arrows from the Standard Uniform to the Exponential distribution (R8C3), Rectangular (R8C8), Loglogistic (R9C5), Logistic (R10C5), and Pareto (R10C3).

To indicate that the inverse cdf of a continuous random variable can be expressed as a closed form, we place a black circle in the right bottom of the corresponding box. Distributions with this property include Weibull (R6C2) and Rayleigh (R7C1). To indicate that the geometric (R1C2 and $\mathrm{R} 2 \mathrm{C} 2$ ) and exponential (R8C3) distributions possess memoryless property, we mark a "a circled x" in the left bottom of these three entries.

The relation of any cell to itself is indicated by a connection to itself. Such "self-directed" connections show eight relations; $\mathrm{C}, \mathrm{C}^{\mathrm{iid}}, \mathrm{I}, \mathrm{L}, \mathrm{M}_{\mathrm{n}}, \mathrm{M}_{\mathrm{x}}, \mathrm{P}$, and $\mathrm{S}$. (The notation is defined below in alphabetical order.) It should be noted that the parameters of source and target distributions for the self-directed connections are not the same.

1. The convolution property (C).

The target random variable is ${ }_{i=1}^{n} X_{i}$ where $X_{i}, i=1,2, \ldots, n$, are independent source random variables. For example, the self-directed connection on Chi-Squared (R4C4) indicates that the sum of $n$ independent chi-square random variables yields a new random variable following chi-square distribution.

2. The convolution property requiring iid source random variables $\left(\mathrm{C}^{\mathrm{iid}}\right)$.

The target random variable is ${ }_{i=1}^{n} X_{i}$, where $X_{i}, i=1,2, \ldots, n$, are iid source random variables. For example, the self-directed connection on Cauchy (R4C8) indicates that the sum of $n$ iid Cauchy random variables yields a new random variable following Cauchy distribution. It should be noted that Cauchy (R4C8) is the only one having the $\mathrm{C}_{\mathrm{v}}^{\mathrm{iid}}$ property.

3. The linear combination property $(\mathrm{L})$.

The target random variable is ${ }_{i=1}^{n} a_{i} X_{i}$, where $X_{i}, i=1,2, \ldots, n$, are independent source random variables and $a_{i}$ are real constants. For example, the self-directed connection from Normal ( $\mathrm{R} 4 \mathrm{C} 2$ ) back to the normal indicates that the linear combination of independent normal random variables yields a normal distribution.

4. The inverse property (I).

The target random variable is $\frac{1}{X}$, where $X$ is the source random variable. For example, the self-directed connection on F (R4C5) indicates that the inverse of an F random variable follows F distribution.

5. The product property $(\mathrm{P})$.

The target random variable is ${ }_{i=1}^{n} X_{i}$, where $X_{i}, i=1,2, \ldots, n$, are independent source random variables. For example, the self-directed connection on Log Normal (R5C2) indicates that the product of $n$ independent Log Normal random variables yields a new random variable following Log Normal distribution.

6. The scaling property $(\mathrm{S})$.

The target random variable is $k X$, where $X$ is the source random variable and $k$ is a positive real constant. For example, the self-directed connection on Exponential (R8C3) indicates that $k$ times an independent exponential random variable yields a new random variable following exponential distribution.

7. The minimum property $\left(\mathrm{M}_{\mathrm{n}}\right)$.

The target random variable is $\min \left(X_{1}, X_{2}, \ldots, X_{n}\right)$, where $X_{i}, i=1,2, \ldots, n$, are iid source random variables. For example, the self-directed connection on Exponential (R8C3) indicates that the smallest of $n$ iid exponential random variables follows the exponential distribution. 
8. The maximum property $\left(\mathrm{M}_{\mathrm{x}}\right)$.

The target random variable is $\max \left(X_{1}, X_{2}, \ldots, X_{n}\right)$, where $X_{i}, i=1,2, \ldots, n$, are iid source random variables. For example, the self-directed connection on Standard Power (R9C6) indicates that the largest of $n$ iid standard power random variables follows standard power distribution.

If more than one source random variable is used to form the corresponding target random variable in self-directed connections, independence is required, but iid is not necessary. Specifically, L, C, and $P$ require independence, not necessarily iid, for source random variables. However, $C^{\text {iid }}, M_{x}$, and $M_{n}$ require iid source random variables. Since $L$ implies $C$ and $S$, the $C$ and $S$ properties are not listed on self-directed connections having $\mathrm{L}$ in Figures 1 and 2.

\subsection{The organizing principles}

The organizing principles for defining the ten-by-eight matrix are (1) no connection crossings, (2) shortening the length of connections, (3) grouping distributions with similar properties, and (4) placing alternative forms (e.g., discrete vs. continuous distribution, central vs. non-central, and standard vs non-standard) of the same distributions near each other. The resulting matrix is not unique. There are clearly many other formats that may be more useful depending on other desired organizing principles.

\section{A Simplified Five-by-Five Matrix}

An updated version of an earlier form (see Song 2005) of the matrix format is illustrated in Figure 2, which displays 25 probability distributions in a five-by-five matrix. Four changes have been made: (1) the discrete vs. continuous distributions are made distinct by displaying discrete distributions in rectangular boxes, (2) additional properties (C, C $\mathrm{Cid}, \mathrm{I}, \mathrm{M}_{\mathrm{n}}, \mathrm{M}_{\mathrm{x}}, \mathrm{P}$, and $\left.\mathrm{S}\right)$ related to "self-directed" connections are added, and (3) closed-form and memoryless properties are now marked in their corresponding boxes by, respectively, a black circle in the bottom right and a circled $\mathrm{x}$ in the bottom left. To avoid confusion with index numbers used in Appendix A, we do not list row and column numbers in Figure 2. This simpler version is valuable because it covers only the distributions that are taught in introductory courses and used by most practitioners in industrial settings.

\section{ACKNOWLEDGMENTS}

This paper is based upon work supported by the National Science Council under Grant No. NSC-902218-E-007-018. The authors deeply thank Dennis Engi and Bruce Schmeiser for their perceptive comments.

\section{A MASS AND DENSITY FUNCTIONS}

To define the notation used in Figures 1 and 2, we state the mass and density functions here. The usual "and zero, elsewhere" is implied.

\section{Discrete Distributions}

1. Benford (R3C8):

$$
f_{X}(x)=\log _{10}\left(1+\frac{1}{x}\right), x=1,2, \ldots, 9
$$

2. Bernoulli $(p)(\mathrm{R} 2 \mathrm{C} 5)$ :

$$
f_{X}(x)=p^{x}(1-p)^{1-x}, x=0,1
$$

3. Beta Binomial $(n, 1,2)(\mathrm{R} 2 \mathrm{C} 7)$ :

$$
\begin{aligned}
& f_{X}(x)=\frac{(n+2)(1+2)(1+x)(n-x+2)}{(n+1)(1)(2)(x+1)(n-x+1)(n+1+2)}, \\
& x=0,1, \ldots, n
\end{aligned}
$$

4. Beta Pascal $(k, 1,2)(\mathrm{R} 2 \mathrm{C} 1)$ : 


$$
f_{X}(x)=C_{x-1}^{k-1} \frac{\mathrm{B}(1+k, x+2-k)}{\mathrm{B}(1,2)}, x=0,1, \ldots
$$

5. Binomial $(n, p)(\mathrm{R} 3 \mathrm{C} 5)$ :

$$
f_{X}(x)=C_{x}^{n} p^{x}(1-p)^{n-x}, x=0,1, \ldots, n
$$

6. Discrete Uniform $\left(x_{1}, x_{2}, \ldots, x_{n}\right)$ (R1C7):

$$
f_{X}(x)=\frac{1}{n}, x=x_{1}, x_{2}, \ldots, x_{n}
$$

7. Discrete Weibull $(p, \quad)(\mathrm{R} 1 \mathrm{C} 1)$ :

$$
f_{X}(x)=(1-p)^{x}-(1-p)^{(x+1)}, x=0,1, \ldots
$$

8. Equal-Spaced Uniform $(a, b, c)$ (R1C8):

$$
f_{X}(x)=\left(1+\frac{b-a}{c}\right)^{-1}, x=a, a+c, a+2 c, \ldots, b
$$

9. Gamma Poisson ( , ) (R3C3):

$$
f_{X}(x)=\frac{(x+)^{x}}{()(1+)^{+x} x !}, x=0,1, \ldots
$$

10. Geometric(trials) $(p)(\mathrm{R} 2 \mathrm{C} 2)$ :

$$
f_{X}(x)=p(1-p)^{x-1}, x=1,2, \ldots
$$

11. Geometric(failures) $(p)$ (R1C2):

$$
f_{X}(x)=p(1-p)^{x}, x=0,1, \ldots
$$

12. Hypergeometric $(N, K, n)$ (R2C6):

$$
f_{X}(x)=\frac{C_{x}^{K} C_{n-x}^{N-K}}{C_{n}^{N}}, x=\max \{n-N+K, 0\}, \ldots, \min \{K, n\}
$$

13. Logarithm $(c)$ (R1C3):

$$
f_{X}(x)=\frac{-(1-c)^{x}}{x \ln c}, x=1,2, \ldots
$$

14. Negative Binomial, Pascal $(k, p)(\mathrm{R} 3 \mathrm{C} 2)$ :

$$
f_{X}(x)=C_{k-1}^{x-1} p^{k}(1-p)^{x-k}, x=k, k+1, \ldots
$$

15. Negative Hypergeometric $(N, K, k)(\mathrm{R} 2 \mathrm{C} 3)$ :

$$
f_{X}(x)=\frac{C_{k-1}^{K} C_{x-k}^{N-K}}{C_{x-1}^{N}} \cdot \frac{K-k+1}{N-x+1}, x=k, k+1, \ldots, N-K+k
$$

16. Poisson ( ) (R3C4):

$$
f_{X}(x)=\frac{{ }^{x} \mathrm{e}^{-}}{x !}, x=0,1,2, \ldots
$$

17. Polya $(n, p, \quad)$ (R1C4):

$$
\begin{aligned}
& f_{X}(x)=\frac{C_{x}^{n} \quad \begin{array}{c}
x-1 \\
j=0
\end{array}(p+j) \cdot \begin{array}{c}
n-x-1 \\
k=0
\end{array}(1-p+k)}{n-1}(1+i) \\
& x=0,1,2, \ldots, n
\end{aligned}
$$

18. Power Series $(c, A(c))$ (R2C4):

$$
f_{X}(x)=\frac{a_{x} c^{x}}{A(c)}, \text { where } c>0 \text { and } A(c)={ }_{x} a_{x} c^{x}, x=0,1,2, \ldots
$$

19. Standard Discrete Uniform $(0, n, 1)(\mathrm{R} 2 \mathrm{C} 8)$ :

$$
f_{X}(x)=\frac{1}{n+1}, x=0,1,2, \ldots, n
$$

20. Zeta ( ) (R1C5): 
$f_{X}(x)=\frac{1}{x_{i=1}(1 / i)}, x=0,1,2, \ldots$

21. Zipf $(n, \quad)$ (R1C6):

$$
f_{X}(x)=\frac{1}{x{ }_{i=1}^{n}(1 / i)}, x=1,2, \ldots, n
$$

\section{Definition of Continuous Distributions}

1. Arcsine (R6C7):

$$
f_{X}(x)=[\sqrt{x(1-x)}]^{-1}, 0<x<1
$$

2. $\operatorname{Beta}(1,2)$ (R7C5):

$$
f_{X}(x)=\frac{(1+2) x^{1^{-1}}(1-x)^{2^{-1}}}{(1)(2)}, 0<x<1
$$

3. Cauchy $(, \quad)$ (R4C8):

4. Chi ( ) (R5C4):

$$
f_{X}(x)=\left\{\left[1+\left(\frac{x-}{-}\right)^{2}\right]\right\}^{-1},-<x<
$$

$$
f_{X}(x)=\frac{x^{-1} \mathrm{e}^{-x^{2} / 2}}{(/ 2) 2^{/ 2-1}}, x>0
$$

5. Chi-Squared ( ) (R4C4):

$$
f_{X}(x)=\frac{x^{/ 2-1} \mathrm{e}^{-x / 2}}{(/ 2) 2^{/ 2}}, x>0
$$

6. Doubly Noncentral F ( $1,2, \quad, \quad)$ (R5C7):

$$
\begin{aligned}
& f_{X}(x)={ }_{j=0 k=0}\left[\frac{e^{-/ 2}\left(\frac{1}{2}\right)^{j}}{j !}\right]\left[\frac{e^{-/ 2}\left(\frac{1}{2}\right)^{k}}{k !}\right] \\
& \times \quad(1 / 2)+j \quad(2 / 2)+k{ }_{1}\left({ }^{1 / 2}\right)+j-1 \\
& \times\left(2+{ }_{1} x\right)^{-\frac{1}{2}\left(1_{1}+2\right)-j-k}
\end{aligned}
$$

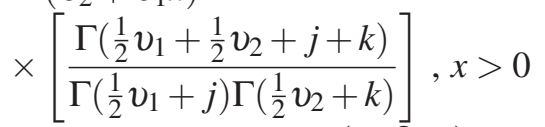

7. Doubly Noncentral $\mathrm{T}(\mathrm{,}, \quad)$ (R5C8):

See Johnson, Kotz, and Balakrishnan (1995, p.533)

8. Erlang $(k, \quad)(\mathrm{R} 7 \mathrm{C} 4)$ :

$$
f_{X}(x)=\frac{x^{k-1} \exp (-x /)}{k(k-1) !}, x>0
$$

9. $\operatorname{Error}(m, 1,2)(\mathrm{R} 10 \mathrm{C} 1)$ :

$$
f_{X}(x)=\frac{\exp \left[-\left(|x-m| / 1_{1}\right)^{2 / 2} / 2\right]}{{ }_{1}\left(22^{2 / 2+1}(1+2 / 2)\right)},-<x<
$$

10. Exponential ( ) (R8C3):

$$
f_{X}(x)=-1 \exp (-x /), x>0
$$

11. Exponential Power ( , ) (R9C4):

$$
f_{X}(x)=\exp [1-\exp (x)] \exp (x) \quad x^{-1}, x>0
$$

12. Extreme Value ( , ) (R6C1):

$$
f_{X}(x)=(/) \exp [x-\exp (x) /],-<x<
$$

13. F ( 1,2$)$ (R4C5): 


$$
f_{X}(x)=\frac{\left(\frac{1+2}{2}\right)(1 / 2)^{1 / 2}}{(1 / 2)(2 / 2)} \frac{x^{(1 / 2)-1}}{\left(1+12^{-1} x\right)^{(1+2) / 2}}, x>0
$$

14. Gamma ( , ) (R6C4):

$$
f_{X}(x)=\frac{(x /)^{-1} \exp (-\mathrm{x} /)}{()}, x>0
$$

15. Gamma Normal ( , , ) (R5C1):

See Evans, Hastings, and Peacock (2000, p.103)

16. Generalized Gamma ( $1, \quad 2, \quad$ ) (R7C3):

$$
f_{X}(x)=\frac{2}{(1)}\left(\frac{x}{-}\right)^{12^{-1}} \exp \left[-\left(\frac{\mathrm{x}}{-}\right)^{2}\right], x>0
$$

17. Generalized Pareto ( $1,2, \quad)(\mathrm{R} 10 \mathrm{C} 2)$ :

$$
f_{X}(x)=\left(1+\frac{2}{x+}\right)\left(1+\frac{x}{-}\right)^{-2} \exp (-1 x), x>0
$$

18. Gompertz $(k, \quad)$ (R9C7):

$$
f_{X}(x)=k^{x} \exp \left[-k\left({ }^{x}-1\right) / \ln \right], x>0
$$

19. Hyperbolic Secant (R3C7):

$$
f_{X}(x)=\operatorname{sech}(x),-<x<
$$

20. Hyperexponential ( $i, i=1,2, \ldots, n)(\mathrm{R} 7 \mathrm{C} 2)$ :

$$
f_{X}(x)={ }_{i=1}^{n} \frac{p_{i}}{i} e^{-x / i} \text {, where } p_{i}>0 \text { and }{ }_{i=1}^{n} p_{i}=1, x>0
$$

21. Hypoexponential $(i, i=1,2, \ldots, n)(\mathrm{R} 9 \mathrm{C} 2)$ :

$$
f_{X}(x)={ }_{i=1}^{n}(1 / i) e^{-x / i}\left(c_{j=1, j \neq i}^{n} \frac{i}{i-j}\right) \text {, where } i \neq j, \forall i \neq j, x>0
$$

22. Increasing Decreasing Bathtub, $\operatorname{IDB}(k, 1,2)(\mathrm{R} 8 \mathrm{C} 1)$ :

$$
f_{X}(x)=\frac{\left(1+{ }_{2} x\right) k x+1}{\left(1+{ }_{2} x\right)^{1 / 2+1}} \exp \left(-k x^{2} / 2\right), x>0
$$

23. Inverse Gaussian, Wald ( , ) (R4C1):

$$
f_{X}(x)=\sqrt{\frac{}{2 x^{3}}} \exp \left[-\frac{}{22^{2} x}(x-)^{2}\right], x>0
$$

24. Inverted Beta $(1,2)$ (R7C7):

$$
f_{X}(x)=\frac{x^{2^{-1}}(1+x)^{-1} 1^{-2}}{\mathrm{~B}(1,2)}, x>0
$$

25. Inverted Gamma ( , ) (R6C5):

$$
f_{X}(x)=\frac{1}{()} x^{--1} \exp [-1 /(x)], x>0
$$

26. Kolmogorov-Smirnov ( $n$ ) (R6C8):

See Drew,Glen, and Leemis(2000)

27. Laplace( , ) (R9C1):

$$
f_{X}(x)=(2)^{-1} \exp [-|\mathrm{x}-\quad| / \quad],-<x<
$$

28. Lévy (R6C7):

$$
f_{X}(x)=\sqrt{-} \frac{e^{-/ 2 x}}{x^{3 / 2}}, x>0
$$

29. Log Gamma ( , ) (R5C3):

$$
f_{X}(x)=\frac{1}{()} \exp (x) \exp \left(-\mathrm{e}^{x} / \quad\right),-<x<
$$


30. Log Logistic ( $1, \quad 2)$ (R9C5):

$$
f_{X}(x)=\frac{{ }_{2} \mathrm{e}^{-1} x^{-2-1}}{\left[1+\mathrm{e}^{-1} x^{-2}\right]^{2}}, x>0
$$

31. Log Normal $(m, \quad)$ (R5C2):

$$
f_{X}(x)=\left(\begin{array}{ll}
x & \sqrt{2}
\end{array}\right)^{-1} \exp \left[-\frac{1}{2}[\underline{\ln (x / m)}]^{2}\right], x>0
$$

32. Logistic ( , ) (R10C5):

$$
f_{X}(x)=\frac{\exp [-(\mathrm{x}-) /]}{\{1+\exp [-(\mathrm{x}-) /]\}^{2}},-<x<
$$

33. Logistic Exponential ( , ) (R9C3):

$$
f_{X}(x)=\frac{(1 /)\left(\mathrm{e}^{x /}-1\right)^{-1} \mathrm{e}^{x /}}{\left(1+\left(\mathrm{e}^{x /}-1\right)\right)^{2}}, x>0
$$

34. Lomax ( , ) (R10C6):

$$
f_{X}(x)=\frac{}{(1+x)^{+1}}, x>0
$$

35. Makeham $(k, \quad 1, \quad 2)$ (R9C8):

$$
f_{X}(x)=\left(\begin{array}{ll}
1+k & \underset{2}{x}
\end{array}\right) \exp \left[-1 x-\frac{k\left(\begin{array}{l}
x \\
2
\end{array}-1\right.}{\ln 2}\right], x>0
$$

36. Minimax $(1, \quad 2)(\mathrm{R} 10 \mathrm{C} 8)$ :

$$
f_{X}(x)=12 x^{2-1}\left(1-x^{2}\right)^{1-1}, 0<x<1
$$

37. Muth ( ) (R8C2):

$$
f_{X}(x)=\left(\mathrm{e}^{x}-\right) \exp \left[-\frac{1}{\left.\mathrm{e}^{x}+x+\frac{1}{-}\right], x>0}\right.
$$

38. Nakagami $(k, \quad)$ (R6C3):

$$
f_{X}(x)=\frac{2}{() k} x^{2}-1 \exp \left(-\frac{-}{k} x^{2}\right), x>0
$$

39. Noncentral Beta ( $1,2, \quad)$ (R7C6):

$$
\begin{aligned}
& f_{X}(x)=_{i=0} \frac{(i+2+1)}{(1)(i+2)}\left(\frac{e^{-/ 2}}{i !}\right)\left(\frac{1}{2}\right)^{i} x^{i+}{ }^{-1}(1-x){ }^{1-1}, \\
& 0<x<1
\end{aligned}
$$

40. Noncentral Chi-Squared ( , ) (R5C5):

$$
f_{X}(x)={ }_{k=0} \frac{\mathrm{e}^{-/ 2}(/ 2)^{k}}{k !} \cdot \frac{\mathrm{e}^{-x / 2} x(+2 k) / 2-1}{2(+2 k) / 2[(+2 k) / 2]}, x>0
$$

41. Noncentral F ( $1,2, \quad)$ (R5C6):

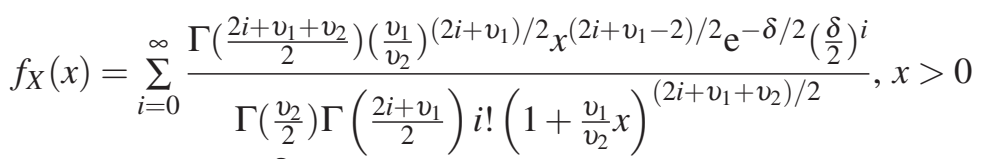

42. Noncentral T ( , ) (R4C7):

$$
\begin{aligned}
& f_{X}(x)=\frac{12 \mathrm{e}^{-{ }^{2} / 2}}{\sqrt{ }(/ 2)\left(+x^{2}\right)^{(+1) / 2}} \underset{i=0}{-\quad<x<} \\
& -\quad \frac{[(+i+1) / 2]}{i !}\left(\frac{x \sqrt{2}}{\sqrt{+x^{2}}}\right)^{i},
\end{aligned}
$$

43. Normal, Gaussian $\left({ }^{,}{ }^{2}\right)(\mathrm{R} 4 \mathrm{C} 2)$ :

$$
f_{X}(x)=\frac{1}{\sqrt{2}} \exp \left[-\frac{1}{2}\left(\frac{x-}{2}\right)^{2}\right],-<x<
$$


44. Pareto ( , ) (R10C3):

$$
f_{X}(x)=\frac{}{x+1}, x \geq
$$

45. Power ( , ) (R10C7):

$$
f_{X}(x)=\frac{x^{-1}}{}, 0<x<
$$

46. Rayleigh ( ) (R7C1):

$$
f_{X}(x)=\left(2 x /{ }^{2}\right) \exp \left[-(x /)^{2}\right], x>0
$$

47. Rectangular, Uniform $(a, b)$ (R8C8):

$$
f_{X}(x)=1 /(b-a), a<x<b
$$

48. Standard Cauchy (R3C6):

$$
f_{X}(x)=\left[\left(1+x^{2}\right)\right]^{-1},-<x<
$$

49. Standard Logistic (R10C4):

$$
f_{X}(x)=\frac{\exp (-x)}{[1+\exp (-x)]^{2}},-\quad<x<
$$

50. Standard Normal (R4C3):

$$
f_{X}(x)=\frac{1}{\sqrt{2}} \exp \left(-x^{2} / 2\right),-<x<
$$

51. Standard Power ( ) (R9C6):

$$
f_{X}(x)=x^{-1}, 0<x<1
$$

52. Standard Triangular (R8C5):

$$
f_{X}(x)= \begin{cases}x+1, & -1<x<0 \\ 1-x, & 0 \leq x<1\end{cases}
$$

53. Standard Uniform (R8C4):

$$
f_{X}(x)=1,0<x<1
$$

54. Standard Wald ( ) (R3C1):

$$
f_{X}(x)=\sqrt{\frac{2 x^{3}}{2}} \exp \left[-\frac{1}{2 x}(x-1)^{2}\right], x>0
$$

55. T ( ) (R4C6):

$$
f_{X}(x)=\frac{[(+1) / 2]}{(/ 2) \sqrt{ }}\left(1+\frac{x^{2}}{-(+1) / 2},-<x<\right.
$$

56. Triangular $(a, m, b)$ (R8C6):

$$
f_{X}(x)= \begin{cases}\frac{2(x-a)}{(m-a)(b-a)}, & a<x \leq m \\ \frac{2(b-x)}{(b-a)(b-m)}, & m<x<b\end{cases}
$$

57. Two-Sided Power, TSP $(a, b, m, n)$ (R8C7):

$$
f_{X}(x)= \begin{cases}\frac{n}{b-a}\left(\frac{x-a}{m-a}\right)^{n-1} & a<x \leq m \\ \frac{n}{b-a}\left(\frac{b-x}{b-m}\right)^{n-1} & m<x<b\end{cases}
$$

58. von Mises ( , ) (R7C8):

$$
f_{X}(x)=\frac{\exp [\cos (x-)]}{2 I_{0}()}, \text { where } I_{0}(\quad)={ }_{i=0} \frac{2 i}{2^{2 i}(i !)^{2}}, 0<x<2
$$

59. Weibull ( , ) (R6C2):

$$
f_{X}(x)=-x^{-1} \exp [-(x /)], x>0
$$




\section{REFERENCES}

Devroye, L. 2006. Nonuniform Random Variate Generation, in Simulation, eds. S.G. Henderson and B.L. Nelson.Handbooks in Operations Research and Management Science. Amsterdam: North Holland.

Dudewicz, E. and Z. Karian 2009. Handbook of Fitting Statisitical Distributions with R. Chapman and Hall.

Evans, M., N. Hastings and B. Peacock 2000. Statistical Distributions (3rd ed.). New York: Wiley.

Gentle, J. E. 2003. Random Number Generation and Monte Carlo Methods. New York: SpringerVerlog.

Johnson, N. L. and S. Kotz 1970. Distributions in Statistics. New York: John Wiley \& Sons.

Johnson, N. L., S. Kotz and N. Balakrishnan 1995. Continuous Univariate Distributions (Vol. II, 2nd ed.). New York: Wiley.

Kotz, S. and J. R. van Dorp 2004. Beyond Beta: Other Continuous Families of Distributions with Bounded Support and Applications. World Scientific Publishing Company.

Leemis, L. 1986. Relationships Among Common Univariate Distributions. The American Statistician 40:143-146.

Leemis, L. and J. McQueston 2008. Univariate Distribution Relationships. The American Statistician 62:45-53.

Nakagawa, T. and H. Yoda 1977. Relationships Among Distributions. IEEE Transactions on Reliability 26: 352-353.

Patil, G. P., M. T. Boswell, S. W. Joshi and M. V. Ratnaparkhi 1985. Discrete Models. Burtonsville: International Co-operative Publishing House.

Patil, G. P., M. T. Boswell and M. V. Ratnaparkhi 1985. Univariate Continuous Models. Burtonsvill: International Co-operative Publishing House.

Schmeiser, B. W. 1980. Random Variate Generation: A Survey, in Simulation with Discrete Models: A State-of-the-Art View, ed. T. I. Oren, C. M. Shub, and P. F. Roth. Institute of Electrical and Electronic Engineers. New York.

Song, W. T. 2005. Relationships Among Some Univariate Distributions. IIE Transactions 37: 651-656.

\section{AUTHOR BIOGRAPHIES}

WHEYMING T. SONG is a professor in the Department of Industrial Engineering at the National Tsing Hua University in Taiwan. She received her undergraduate degree in statistics and masters degree in industrial engineering at Cheng-Kung University in Taiwan in 1979. She then received masters degrees in applied mathematics in 1983 and industrial engineering in 1984, both from the University of Pittsburgh. Dr. Song received her Ph.D. from the School of Industrial Engineering at Purdue University in 1989. She Joined Tsing Hua in 1990 after spending one year as a visiting assistant professor at Purdue IE. Her research interests are applied operations research; probability and statistics; and the statistical aspects of stochastic simulation, including input modeling, output analysis, variance reduction, and ranking and selection. Her email is: <wheyming@ie.nthu .edu.tw>.

YI-CHUN CHEN is a Ph.D student in the Department of Industrial Engineering at the National Tsing Hua University in Taiwan. He received his undergraduate degree in mathematics at National Central University in 1999 and masters degree in industrial engineering at National Taiwan University in 2001

in Taiwan. His research interests are statistical quality control, simulation, and system reliability. His email address is <d937817@oz.nthu.edu.tw $>$. 